\title{
Fractional erbium-doped yttrium aluminum garnet laser-assisted drug delivery of hydroquinone in the treatment of melasma
}

This article was published in the following Dove Press journal:

Clinical, Cosmetic and Investigational Dermatology

\author{
Ashraf M Badawi ${ }^{1-4}$ \\ Mai Abdelraouf Osman' \\ 'Dermatology Unit, Department of \\ Medical Applications of Lasers (MAL), \\ National Institute of Laser Enhanced \\ Sciences (N.I.L.E.S.), Cairo University, \\ Giza, Egypt; ${ }^{2}$ Dermatology and Laser \\ Applications, Szeged University, \\ Szeged, Hungary; ${ }^{3}$ European Society \\ for Lasers and Energy Based Devices, \\ Strasbourg, France; ${ }^{4}$ European \\ Society for Cosmetic and Aesthetic \\ Dermatology, Paris, France
}

Background: Melasma is a difficult-to-treat hyperpigmentary disorder. Ablative fractional laser (AFL)-assisted delivery of topically applied drugs to varied targets in the skin has been an area of ongoing study and research.

Objective: The objective of this study was to evaluate the efficacy and safety of fractional erbium-doped yttrium aluminum garnet (Er:YAG) laser as an assisted drug delivery for enhancing topical hydroquinone (HQ) permeation into the skin of melasma patients.

Patients and methods: Thirty female patients with bilateral melasma were randomly treated in a split-face controlled manner with a fractional Er:YAG laser followed by $4 \%$ HQ cream on one side and 4\% HQ cream alone on the other side. All patients received six laser sessions with a 2-week interval. The efficacy of treatments was determined through photographs, dermoscopic photomicrographs and Melasma Area Severity Index (MASI) score, all performed at baseline and at 12 weeks of starting therapy. The patient's level of satisfaction was also recorded.

Results: Er:YAG laser + HQ showed significantly better results $(p<0.005)$ with regard to decrease in the degree of pigmentation as assessed on the 4-point scale than HQ alone. There was a significant decrease in MASI scores on Er:YAG laser + HQ side vs HQ side. Minor reversible side effects were observed on both sides.

Conclusion: AFL-assisted delivery of HQ is a safe and effective method for the treatment of melasma.

Keywords: MASI score, dermoscopy, physician global assessment

\section{Introduction}

Melasma, a name derived from the Greek melas, or black, is one of the most common causes of acquired hypermelanosis of the face. It is characterized by more or less symmetrically distributed, medium- to dark-brown macules with polycyclic or arciform borders, affecting sun-exposed areas, particularly forehead, cheeks, temples, and upper lip. ${ }^{1}$

Although the precise cause of melasma is unknown, multiple factors have been implicated in the etiopathogenesis of this condition, including genetic influences, exposure to ultraviolet radiation and visible light, pregnancy, oral contraceptives, estrogen-progesterone therapies, thyroid dysfunction, cosmetics, and phototoxic and antiseizure drugs. ${ }^{2}$ The chronic and relapsing nature of melasma is an important issue, as any therapeutic results achieved might be difficult to maintain for a long time. ${ }^{3}$ Conventional treatments for melasma include elimination of any possible causative problem and the use of an adequate sunscreen and depigmenting agents, with and
Correspondence: Mai Abdelraouf Osman Dermatology Unit, Department of Medical Applications of Lasers, National Institute of Laser Enhanced Sciences, Cairo University, 25 Giza Street, Giza 12316, Egypt

Tel +2035728221

Email maiosman2007@yahoo.com 
without the combination of other therapies, such as tretinoin, topical corticosteroids, or superficial peeling agents. ${ }^{2,4}$

The most commonly used depigmenting agent for the treatment of melasma is hydroquinone (HQ), which is an aromatic organic compound. It reduces the conversion of L-dopa to melanin by inhibiting the enzyme tyrosinase. ${ }^{5}$

The therapeutic efficacy of topical drugs is related to their ability to penetrate and distribute into skin layers, reaching target cells. The major rate-limiting step for percutaneous absorption is passage through stratum corneum (SC). ${ }^{6}$ Many delivery strategies have been developed to aid drug uptake. Commonly used techniques have included tape stripping to remove the SC, electroporation, iontophoresis, microneedling, and ablative lasers. However, these approaches are limited due to the associated potential pain and irritation. ${ }^{7}$

Ablative fractional laser (AFL) is a relatively new device that relies on the concept of fractional photothermolysis. It delivers controlled thermal injury in an array of microscopic treatment zones (MTZs) composed of vertical columns of ablated tissue surrounded by untreated intact tissue that allow for more rapid reepithelialization and lower risk of scarring. Ablation of the SC allows a molecule to bypass penetration pathways, allowing for enhanced drug delivery. ${ }^{8-10}$ The aim of the current study was to evaluate the efficacy and safety of fractional erbium-doped yttrium aluminum garnet (Er:YAG) laser as a drug delivery of HQ in melasma patients.

\section{Patients and methods}

This study was designed as a single-blinded, controlled, randomized, split-face study. In all, 32 female patients with Fitzpatrick skin phototypes III-V having mild, moderateto-severe bilaterally symmetrical melasma were included in this study. The exclusion criteria included pregnant women, women on contraceptive pills or isotretinoin at the time of the study or in the past 6 months, patients on any concurrent therapy, and patients with hypersensitivity to HQ, concurrent illnesses, or active or recurrent herpes labialis or keloidal tendencies. None of the patients received any treatment for melasma 1 month before study entry. All the patients were selected among those attending the Outpatient Clinic of Dermatology, National Institute of Laser Enhanced Sciences (NILES), Cairo University. The procedure, risks, benefits, potential complications, and side effects of the procedure were explained to all patients, and written informed consent were obtained before commencement of the study, including approval for publishing their photos of before and after treatment. The study protocol conformed to the ethical guidelines of the 1975 Declaration of Helsinki. A documented approval from the Institutional Review Board of NILES, Cairo University, was obtained.

A detailed history was taken before treatment from each patient regarding the etiological factors (sun exposure, cosmetic use, oral contraceptive use or phototoxic drug, past pregnancies, menstrual history, thyroid dysfunction), and family history of melasma was also noted. Wood's lamp examination was performed at the study entry to determine the type of melasma (epidermal, mixed, or dermal).

\section{Treatment protocol}

After gentle cleansing, topical anesthetic cream (EMLA; AstraZeneca plc, Södertälje, Sweden) was applied 60 minutes before intervention over the area to be treated with fractional Er:YAG laser to relieve patient discomfort. Facial halves were randomly assigned to receive treatment with fractional ablative 2,940 nm Er:YAG laser (Fotona Xs Dynamis, Ljubljana, Slovenia) on one side, followed by application of topical $4 \%$ HQ cream. The other side was treated with 4\% HQ cream only as a control. The laser settings were as follows: fluence 250-300 mJ, 30-40 $\mu \mathrm{m}$ ablation depth, handpiece PSO1, spot size $7 \mathrm{~mm}$ in diameter, short pulse (SP) duration mode, MTZ density level of 2-3, and frequency of 4-5 Hz. Two consecutive passes of non-overlapping pulses were done over melasma areas. For the first pass, the laser spots were placed pulse by pulse in a vertical direction, and for the second pass, the spots were placed in a horizontal direction. Parameters for each setting were kept constant in all patients. The handpiece of the laser was held perpendicular to the target tissue. No concurrent use of an epidermal cooling device was performed. Treatments were given in an operating room, and all patients wore protective glasses.

Following treatment, patients were instructed to apply 4\% HQ cream twice daily without rubbing on both sides as a maintenance therapy and a moisturizer (Atoderm PP; Bioderma, Lyon, France) twice daily only if signs of irritation developed. Patients were strictly instructed to avoid excessive sun exposure, apply a broad spectrum sunscreen with a sun protection factor of 50+ in the morning, and reapply the sunscreen every 2 hours. All patients received six treatment sessions at 2-week intervals $(0,2,4,6,8$, and 10 weeks).

\section{Treatment evaluation}

\section{Photography}

Standardized high-resolution digital photographs using identical camera settings (Cyber-shot DSC-HX5V; Sony Corp., Tokyo, Japan) were obtained prior to treatments and 2 weeks after the last session (12 weeks after starting therapy). 


\section{Dermoscopy}

Dermoscopic photomicrographs of the patients were taken at baseline and 2 weeks after the last session using a dermoscope (DermLite П Pro HR; 3 Gen, San Juan Capistrano, CA, USA, magnification $\times 15$ ) It is a palm-sized device that offers high light output with an integrated rechargeable lithium-ion battery and is connected to a camera (Cyber-shot DSC-W710, 16.1 megapixels; Sony Corp.) equipped with an optical zoom $(\times 5)$.

\section{Melasma Area and Severity Index (MASI) score}

Evaluation of MASI scores was performed at baseline and 2 weeks after the end of treatment. The MASI is an index designed to quantify the severity of melasma during therapy, determined by Kimbrough-Green et al. ${ }^{11}$ Briefly, according to the MASI score, the whole face is divided into four areas: the forehead, right malar, left malar, and chin, corresponding to $30 \%, 30 \%, 30 \%$, and $10 \%$ of the total face area, respectively. The grade of melasma severity was determined by three variables: the percentages of total area involved, on a scale of 0 (no involvement) to 6 (90-100\% involvement); darkness, on a scale of 0 (absent) to 4 (maximum); and the homogeneity of hyperpigmentation, on a scale of 0 (minimal) to 4 (maximum). The MASI was then calculated by the following equation:

$$
\begin{aligned}
\text { MASI }= & 0.3\left(\mathrm{D}_{\mathrm{F}}+\mathrm{H}_{\mathrm{F}}\right) \mathrm{A}_{\mathrm{F}}+0.3\left(\mathrm{D}_{\mathrm{MR}}+\mathrm{H}_{\mathrm{MR}}\right) \mathrm{A}_{\mathrm{MR}}+0.3 \\
& \left(\mathrm{D}_{\mathrm{ML}}+\mathrm{H}_{\mathrm{ML}}\right) \mathrm{A}_{\mathrm{ML}}+0.1\left(\mathrm{D}_{\mathrm{C}}+\mathrm{H}_{\mathrm{C}}\right) \mathrm{A}_{\mathrm{C}}
\end{aligned}
$$

where $\mathrm{D}$ is darkness, $\mathrm{H}$ is homogeneity, $\mathrm{A}$ is area, $\mathrm{F}$ is forehead, MR is right malar, ML is left malar, and $\mathrm{C}$ is chin. The values $0.3,0.3,0.3$, and 0.1 are the respective percentages of the total facial area. A high MASI score correlates with severe hyperpigmentation, and this score can be a maximum of up to 48. In this study, MASI score was calculated only for malar area.

\section{Physician Global Assessment (PGA)}

Assessment of clinical improvement was performed by a blinded independent physician who compared pretreatment with posttreatment photographs, photomicrographs, and MASI scores using a quartile grading scale: $>75 \%$ lightening (excellent), $51-75 \%$ (good), 26-50\% (fair), and 0-25\% (poor).

\section{Patient self assessment}

The patient's level of satisfaction was similarly recorded at the end of the study as follows: $>75 \%$ lightening (excellent), $51-75 \%$ (good), $26-50 \%$ (fair), and $0-25 \%$ (poor). Patients were also instructed to report any cutaneous side effects at each postoperative visit and at the end of treatment.

\section{Follow-up}

All the patients were followed up for 12 weeks further after the evaluation visit ( 24 weeks after starting therapy) to detect any recurrence of melasma on both facial sides.

\section{Statistical analyses}

Continuous metric variables were summarized by the mean and SD and were represented graphically using comparative box plots. Categorical variables were summarized by counts (frequencies) and represented graphically by comparative bar charts. For metric variables (MASI score), paired $t$-test was used. For categorical variables, Wilcoxon signed-rank test was used for assessing ordinal variables (PGA and patients' satisfaction), while McNemar's test was used for dichotomous ones (complications). $p$-values $<0.05$ were considered as significant. All analyses were done using IBM SPSS v. 24 (IBM Corporation, Armonk, NY, USA).

\section{Results}

In all, 32 patients with melasma fulfilling the selection criteria were included in the study. A total of 30 patients completed the six treatment sessions. Two patients failed to complete the study and dropped out (one became pregnant and the other one traveled abroad), and they were excluded from the results. Patients' ages ranged from 24 to 52 years (mean $37.87 \pm 7.97$ years), and the duration of melasma varied from 6 months to 13 years (mean $4.68 \pm 3.19$ years). Baseline demographics and clinical data of the patients are listed in Table 1.

According to the physicians' assessment, which was done by comparing the clinical photographs, dermoscopic photomicrographs, and MASI scores of patients at baseline and 12 weeks after starting treatment, both modalities yielded a significant greater improvement in melasma. The side treated with Er:YAG laser + HQ showed significantly better results $(p<0.005)$ with regard to decrease in the degree of pigmentation as assessed on the 4-point scale than the side treated with HQ alone. At the end of treatment sessions, $73.4 \%$ and $40 \%$ of Er:YAG + HQ and HQ sides, respectively, were rated to have $>50 \%$ improvement. The patients' viewpoints on efficacy at the end of the study were similar to physician's evaluation (Tables 2 and 3 and Figures 1 and 2). Dermoscopy of melasma lesions in all studied patients at baseline demonstrated a fine brown reticular pattern superimposed on a background of faint light brown or bluish areas. In addition, telangiectasia was observed in 21 patients (70\%; Figures 3 and 4). 
Table I Baseline demographics and clinical data of the patients

\begin{tabular}{ll}
\hline Characteristic & Value \\
\hline Age (years), mean \pm SD & $37.87 \pm 7.97$ \\
Duration of melasma (years), mean \pm SD & $4.68 \pm 3.19$ \\
Fitzpatrick skin type, $\mathrm{n}(\%)$ & \\
III & II (36.7) \\
IV & $12(40)$ \\
V & $7(23.3)$ \\
Type of melasma, $\mathrm{n}(\%)$ & \\
Epidermal & $15(50)$ \\
Dermal & $6(20)$ \\
Mixed & $9(30)$ \\
Aggravating factors, $\mathrm{n}(\%)$ & \\
Sun exposure & $20(66.7)$ \\
Oral contraceptive pills & $10(33.3)$ \\
Pregnancy & $15(50)$ \\
Cosmetic use & $4(13.3)$ \\
Family history & $13(43.3)$ \\
\hline
\end{tabular}

Table 2 PGA

\begin{tabular}{lll}
\hline PGA & Er:YAG laser + HQ, n (\%) & HQ, $\mathbf{n}(\%)$ \\
\hline Excellent & $8(26.7)$ & $4(13.3)$ \\
Good & $14(46.7)$ & $8(26.7)$ \\
Fair & $6(20)$ & $13(43.3)$ \\
Poor & $2(6.7)$ & $5(16.7)$ \\
\hline
\end{tabular}

Abbreviations: PGA, Physician Global Assessment; Er:YAG, erbium-doped yttrium aluminum garnet; $\mathrm{HQ}$, hydroquinone.

Table 3 Patient evaluation of treatment outcome

\begin{tabular}{lll}
\hline Patient satisfaction & Er:YAG laser + HQ, n (\%) & HQ, n (\%) \\
\hline Excellent & $10(33.3)$ & $5(16.7)$ \\
Good & $12(40)$ & $7(23.3)$ \\
Fair & $7(23.3)$ & $14(46.7)$ \\
Poor & $1(3.3)$ & $4(13.3)$ \\
\hline
\end{tabular}

Abbreviations: Er:YAG, erbium-doped yttrium aluminum garnet; $\mathrm{HQ}$, hydroquinone.

A significant decrease in MASI scores was obtained in both sides at the end of the study. At baseline, the mean MASI scores in the laser + HQ-treated sides and HQ-treated sides were $9.31 \pm 2.84$ and $9.1 \pm 2.70$, respectively, and the difference was not statistically significant $(p=0.171)$. At the end of treatment sessions, the mean MASI scores reached $3.74 \pm 2.86$ in the laser-treated sides and $6.82 \pm 2.97$ in the HQ-treated sides. This difference was statistically significant $(p<0.005$; Figure 5).

Side effects experienced were acceptable by most of the patients and did not necessitate the cessation of treatment. Transient and mild erythema, burning sensation, and itching were reported on both facial sides. All the patients $(100 \%)$ experienced erythema on the laser + HQ-treated side, which

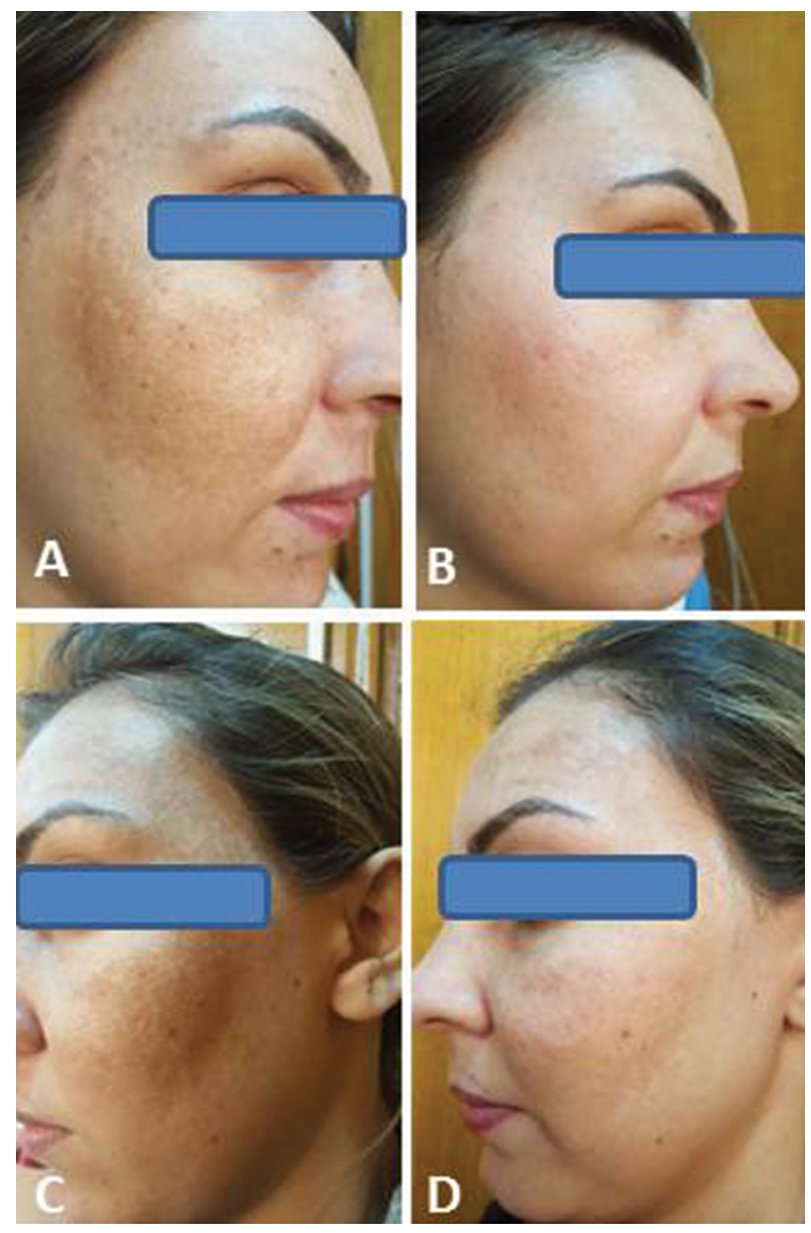

Figure I A 35-year-old female patient with a 4-year history of melasma: (A) before treatment with fractional Er:YAG laser $+\mathrm{HQ}$ cream, (B) clinical appearance 12 weeks after starting therapy, (C) before treatment with $\mathrm{HQ}$ cream alone, and (D) clinical appearance 12 weeks after starting therapy.

Abbreviations: Er:YAG, erbium-doped yttrium aluminum garnet; $H Q$, hydroquinone.

lasted for 3-4 days, while five (16.7\%) patients showed erythema on the HQ-treated side, which lasted for 1-2 days $(p<0.005)$. Superficial crusting subsequently occurred and completely sloughed off in an average of 5 days on the Er:YAG laser side, while no crusting was observed on the HQ-treated side. None of the patients suffered from worsening of melasma on treatment. There was burning sensation in seven (23.3\%) patients with laser + HQ vs five (16.7\%) patients with HQ. There was itching in three patients $(10 \%)$ in each side. These side effects subsided on the application of emollients, and no further intervention was required. Apart from erythema, there was no statistical significant difference between both sides regarding burning sensation and itching ( $p=0.5$ and $p=0.9$, respectively; Table 4). Follow-up period, at 24 weeks from commencing treatment, showed recurrence of melasma lesions in two (6.6\%) patients on both facial sides. 


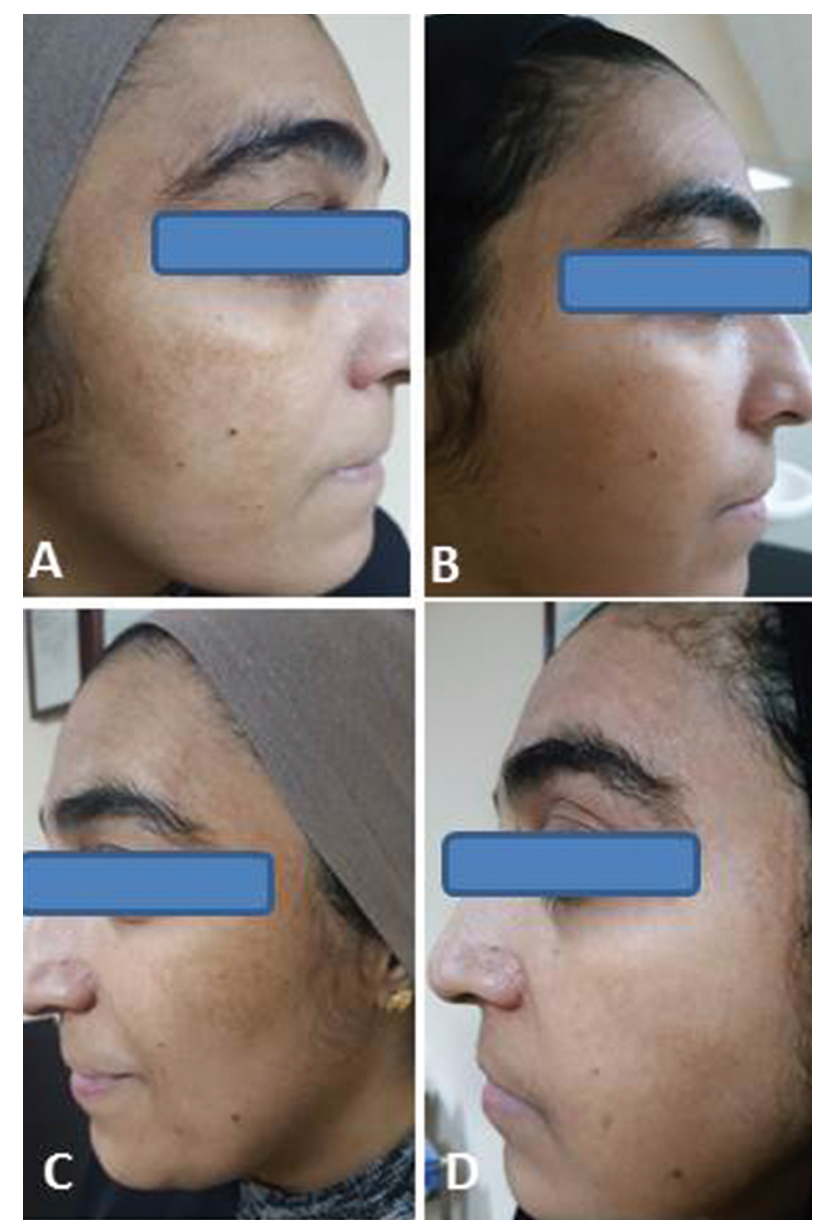

Figure 2 A 43-year-old female patient with a 7-year history of melasma: (A) before treatment with fractional Er:YAG laser + HQ cream, (B) clinical appearance 12 weeks after starting therapy, (C) before treatment with $\mathrm{HQ}$ cream alone, and (D) clinical appearance 12 weeks after starting therapy.

Abbreviations: Er:YAG, erbium-doped yttrium aluminum garnet; $H Q$, hydroquinone.

\section{Discussion}

Despite the wide therapeutic arsenal available for the treatment of melasma, including new to old active topical medications and technologies with lights and peelings, little is said about the potential role of laser as an assisted drug delivery in the treatment of melasma. To our knowledge, our study is the first of its kind to evaluate the feasibility of fractional Er:YAG laser-assisted drug delivery for enhancing the penetration of topical HQ into melasma lesions. This study was designed as a split-face comparison to eliminate individual variability that may confound treatment evaluations.

At the end of treatment sessions, all patients in the current study showed significant improvement on both facial sides. However, significantly better clinical results were observed with fractional Er:YAG laser + HQ than with HQ alone as shown by PGA, patient self-assessment, and MASI score.

Topical drug delivery is essential in dermatology, and the efficacy of topically applied drugs is crucially dependent on their ability to penetrate the outermost skin barrier of SC and epidermis.

Laser therapy is a unique ablative modality that has the ability to destroy the SC, epidermal, and dermal layers of the skin in a predictable and controlled manner, resulting in the potential increased penetration of topically applied drugs. The Er:YAG laser has a wavelength of 2,940 $\mathrm{nm}$ and is strongly absorbed by water in the epidermis. It exerts its ablation effect with minimal penetration depth and minimal heat generation and therefore minimal thermal damage. The $\mathrm{CO}_{2}$ laser has a wavelength of $10,600 \mathrm{~nm}$ and exerts a greater thermal effect
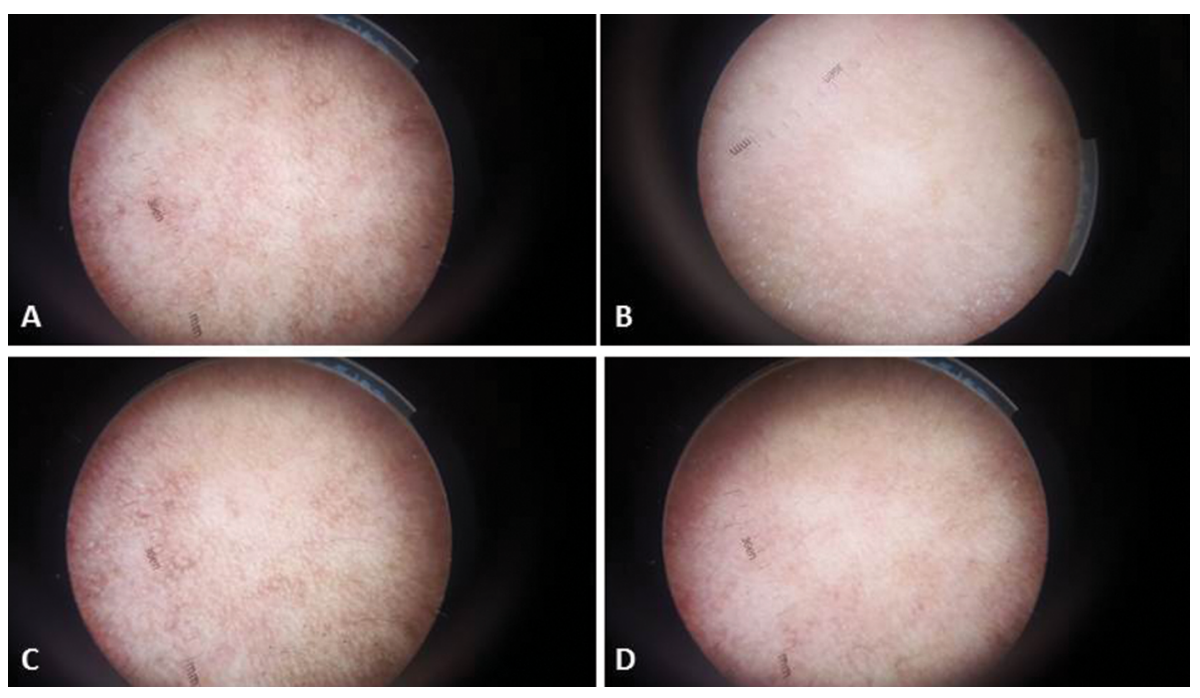

Figure 3 Dermoscopic photomicrographs of melasma lesions shows a fine brown reticular pattern superimposed on a background of faint light brown areas (original magnification $\times 15$ ): (A) at baseline, (B) 12 weeks after starting treatment with fractional Er:YAG laser + HQ cream, (C) at baseline, and (D) I2 weeks after starting treatment with $\mathrm{HQ}$ cream alone.

Abbreviations: Er:YAG, erbium-doped yttrium aluminum garnet; HQ, hydroquinone. 


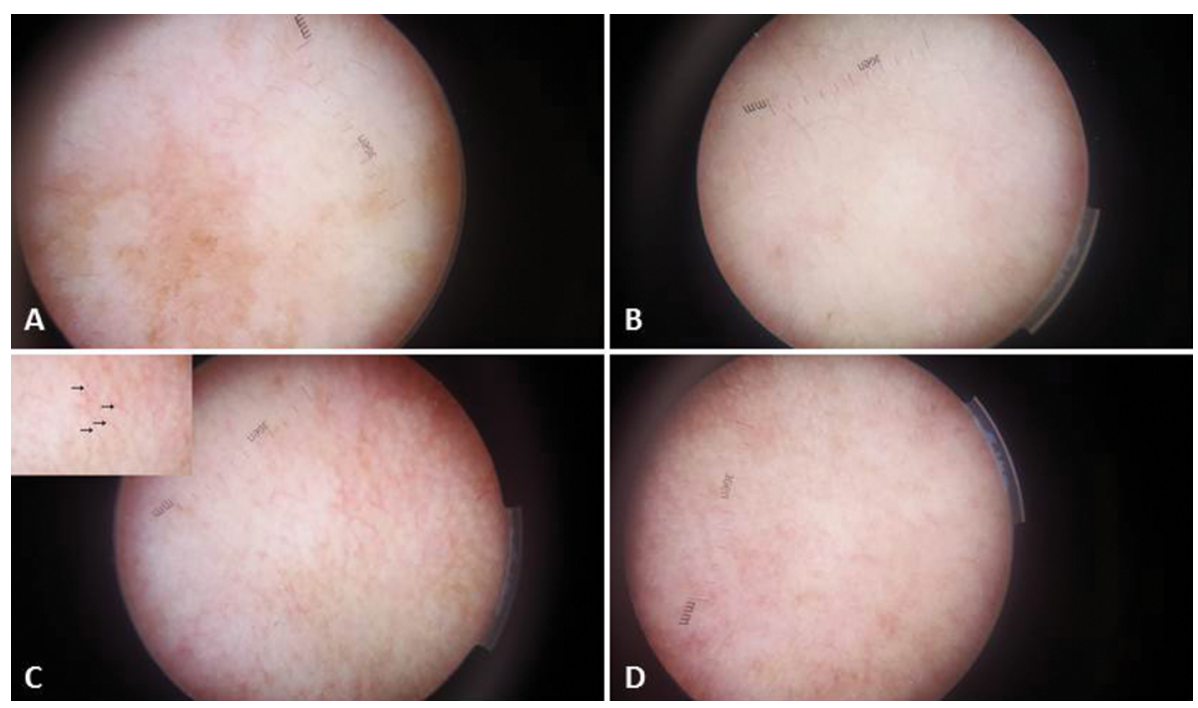

Figure 4 Dermoscopic photomicrographs of melasma lesions (original magnification $\times 15$ ): (A) at baseline, (B) I 2 weeks after starting treatment with fractional Er:YAG laser $+\mathrm{HQ}$ cream, (C) at baseline showing telangiectasias (arrows in inset), and (D) 12 weeks after starting treatment with $\mathrm{HQ}$ cream alone. Abbreviations: Er:YAG, erbium-doped yttrium aluminum garnet; HQ, hydroquinone.

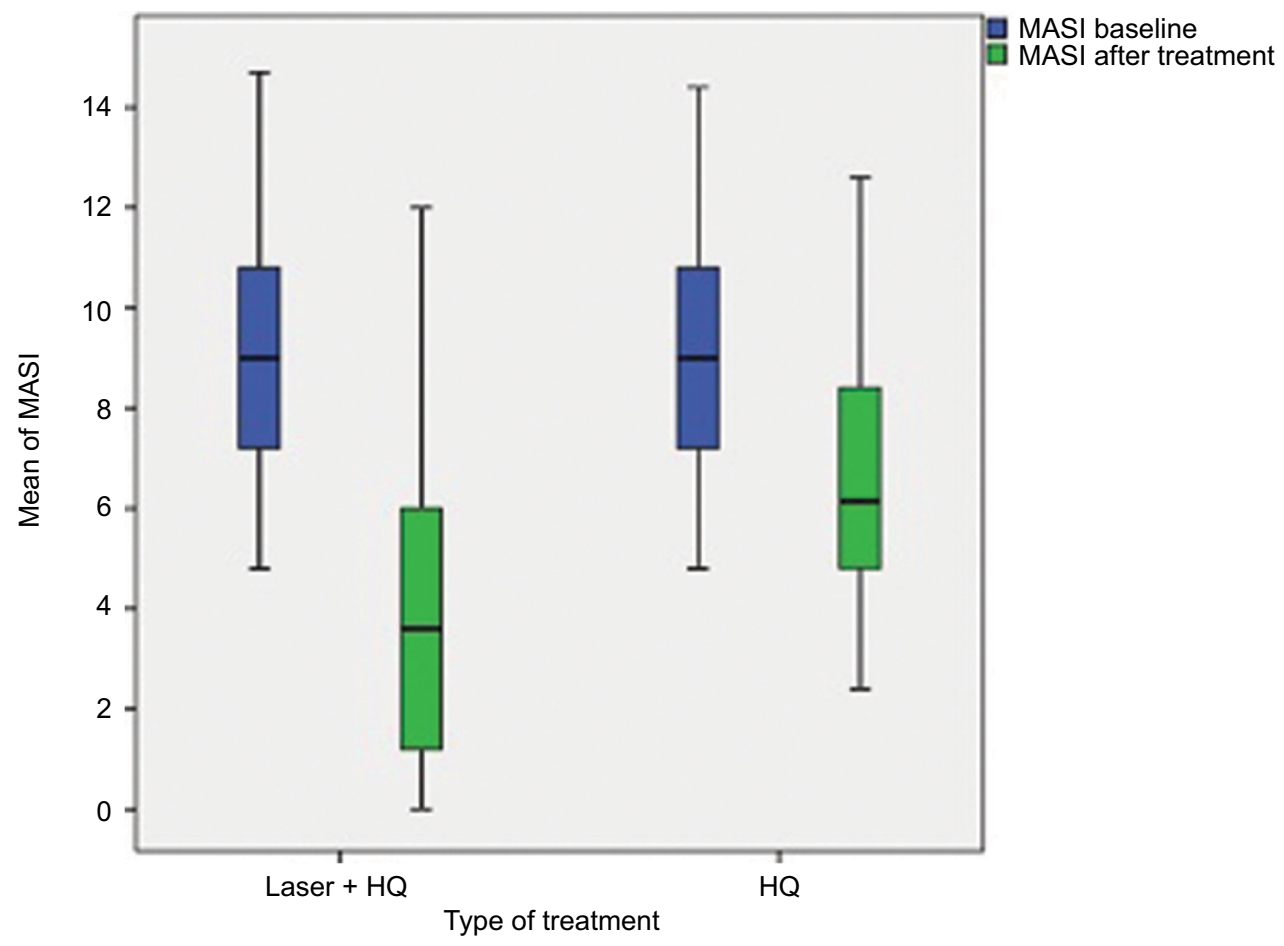

Figure 5 A comparative graph depicting a significant decline in mean MASI score on both sides at the end of the study with a greater reduction on the side receiving fractional Er:YAG laser + HQ than the side receiving HQ alone.

Abbreviations: MASI, Melasma Area Severity Index; Er:YAG, erbium-doped yttrium aluminum garnet; HQ, hydroquinone.

Table 4 Side effects reported

\begin{tabular}{lll}
\hline Side effects & Er:YAG laser + HQ, $\mathbf{n}(\%)$ & HQ, $\mathbf{n}(\%)$ \\
\hline Erythema & $30(100)$ & $5(16.7)$ \\
Itching & $3(10)$ & $3(10)$ \\
Burning sensation & $7(23.3)$ & $5(16.7)$ \\
\hline
\end{tabular}

Abbreviations: Er:YAG, erbium-doped yttrium aluminum garnet; HQ, hydroquinone. than the Er:YAG laser. ${ }^{12,13}$ In 2010, AFL was introduced as a new drug delivery-enhancement technique. ${ }^{14}$ The technique is based on fractional photothermolysis, which can create MTZs of uniform size and depth. These MTZs cross the skin barrier and provide direct access pathways for topically applied drugs to viable epidermis and dermis, thus acting 
as channels for drug uptake until they close by local wound repair without scarring. ${ }^{7,8}$ This could explain the superiority of fractional Er:YAG laser as an assisted drug delivery of HQ over the conventional HQ in the treatment of melasma.

In line with our results, several studies have demonstrated that AFL pretreatment can assist topical drug delivery in a number of skin conditions. Cavalie et $\mathrm{al}^{15}$ studied ablative fractional Er:YAG laser-assisted delivery of topical corticosteroid for treating resistant keloids. A total of 23 patients with 70 keloids were treated with fractional Er:YAG laser, followed by topical application of betamethasone cream twice daily under occlusion. Sessions were performed every other week until complete flattening of the scars was achieved. The median percentage of improvement was $50 \%$, and the median satisfaction of the patients was $7 / 10$. Their study supported the use of laser-assisted delivery of steroids for treating keloid scars.

Another study conducted by Ma et al, ${ }^{16}$ evaluated the feasibility of ablative fractional $\mathrm{CO}_{2}$ laser for enhancing topical timolol permeation into deep infantile hemangiomas. After an average treatment duration of 14.2 weeks, they documented significant regression of hemangiomas according to a global score and the Hemangioma Activity Score. No systemic complications or cutaneous side effects were observed in any of the patients.

A randomized split-face trial was done to assess whether laser-assisted delivery of vitamin C, E, and ferulic topical formula could improve wound healing and decrease postoperative recovery in fractional ablative laser resurfacing for photodamage. The study has shown that vitamin C, E, and ferulic acid correlate with more rapid wound healing post fractional ablative laser and promote the induction of collagen with the ability of patients to return to work and social life more quickly. ${ }^{17}$

Lee et $\mathrm{al}^{18}$ performed a study on porcine and nude mouse skin investigating the ability of a low-fluence fractional Er:YAG laser to increase the transdermal delivery of imiquimod. They demonstrated that a greatly decreased dose of $0.4 \%$ imiquimod with laser pretreatment approximated the delivery of non-pretreated application of 5\% imiquimod.

In another porcine skin model, Hsiao et $\mathrm{al}^{19}$ demonstrated that four or fewer passes with the fractional $\mathrm{CO}_{2}$ laser achieved similar permeation of topical ascorbic acid with less epidermal destruction compared to an equivalent fluence using the conventional $\mathrm{CO}_{2}$ laser.

Similar to fractional lasers, Budamakuntla et $\mathrm{al}^{20}$ stated that microneedling offers an effective and minimally invasive route of drug delivery through the creation of microchannels in the skin. They presented a prospective, randomized study on 60 patients with melasma divided into two groups. Thirty patients were administered with localized microinjections of tranexamic acid (TA) in one group, and other 30 patients were treated with $\mathrm{TA}+$ microneedling at monthly intervals and followed up for 3 consecutive months. In the microinjection group, there was $35.72 \%$ improvement in the MASI score compared to $44.41 \%$ in the microneedling group at the end of third follow-up visit. Six $(26.09 \%)$ patients in the microinjections group, compared to $12(41.38 \%)$ patients in the microneedling group, showed $>50 \%$ improvement.

In the present study, side effects on both sides were comparable. No serious side effects were detected. This could be attributed to the fact that the Er:YAG laser is an excellent ablative tool since its energy is strongly absorbed by watercontaining tissue. This property enables the Er:YAG laser to ablate skin with minimal heat dissipation, thereby potentially minimizing the risk of post-inflammatory hyperpigmentation. ${ }^{21,22}$ Moreover, fractional laser treatment lessens the skin damage caused by traditional ablative lasers and shortens the subsequent recovery period as the undamaged surrounding tissue allows for a reservoir of viable tissue and permits rapid epidermal repair. ${ }^{12,13,23}$ Worthy to be noted, the SP duration used in the present study induces vaporization of the epidermis and the superficial dermis with less collateral thermal damage. A key factor that determines the indirect heating depth is the laser pulse width. If the energy is delivered to the target in a very short time, ablation occurs before significant heat diffusion can take place. This results in less heat being distributed to the surrounding tissue. On the other hand, a long pulse width will allow more heat transfer before ablation takes place, resulting in a greater thermal effect on the surrounding tissue. ${ }^{24}$

A limitation of the present study is the lack of histological assessments of decreased pigmentation and vascular and mast cell changes.

\section{Conclusion}

Based on our findings, fractional Er:YAG laser-assisted transcutaneous delivery of topical HQ is an effective and safe treatment modality for melasma. AFL-assisted drug delivery may become a new important part of both medical and surgical dermatology. Nevertheless, cost will likely be a significant barrier to the widespread adoption of laser-assisted drug delivery techniques, and a cost-benefit analysis should be undertaken by physicians and their patients. As a final note, additional studies are needed to determine the optimal laser parameters in regard to achieve the ideal balance of fluence, treatment density, and drug concentration, ensuring the best efficacy and least incidence of adverse reactions. 


\section{Acknowledgment}

The research was conducted at the NILES.

\section{Disclosure}

The authors report no conflicts of interest in this work.

\section{References}

1. Grimes PE. Melasma: etiologic and therapeutic considerations. Arch Dermatol. 1995;131(12):1453-7.

2. Pandya AG, Guevara IL. Disorders of hyperpigmentation. Dermatol Clin. 2000;18(1):91-98.

3. Rajaratnam R, Halpern J, Salim A, Emmett C. Interventions for melasma. Cochrane Database Sys Rev. 2010;7:CD003583.

4. Ortonne JP, Passeron T. Melanin pigmentary disorders: treatment update. Dermatol Clin. 2005;23(2):209-226.

5. Guevara IL, Pandya AG. Safety and efficacy of $4 \%$ hydroquinone combined with $10 \%$ glycolic acid, antioxidants, and sunscreen in the treatment of melasma. Int J Dermatol. 2003;42(12):966-972.

6. Elias PM, Menon GK. Structural and lipid biochemical correlates of the epidermal permeability barrier. Adv Lipid Res. 1991;24:1-26.

7. Erlendsson AM, Anderson RR, Manstein D, Waibel JS. Developing technology: ablative fractional lasers enhance topical drug delivery. Dermatol Surg. 2014;40:142-146.

8. Manstein D, Herron GS, Sink RK, Tanner H, Anderson RR. Fractional photothermolysis: a new concept for cutaneous remodeling using microscopic patterns of thermal injury. Lasers Surg Med. 2004;34(5):426-438.

9. Alexiades-Armenakas MR, Dover JS, Arndt KA. The spectrum of laser skin resurfacing: nonablative, fractional, and ablative laser resurfacing. J Am Acad Dermatol. 2008;58(5):719-37; quiz 38-40.

10. Brightman LA, Brauer JA, Anolik R, et al. Ablative and fractional ablative lasers. Dermatol Clin. 2009;27(4):479-489.

11. Kimbrough-Green CK, Griffiths CEM, Finkel LJ, et al. Topical retinoic acid (tretinoin) for melasma in black patients- a vehicle controlled clinical trial. Arch Dermatol. 1994;130(6):727-733.
12. Paasch U, Haedersdal M. Laser systems for ablative fractional resurfacing. Expert Rev Med Devices. 2011;8(1):67-83.

13. Waibel J, Beer K, Narurkar V, Alster T. Preliminary observations on fractional ablative resurfacing devices: clinical impressions. J Drugs Dermatol. 2009;8(5):481-485.

14. Haedersdal M, Sakamoto FH, Farinelli WA, Doukas AG, Tam J, Anderson RR. Fractional $\mathrm{CO}_{2}$ laser-assisted drug delivery. Lasers Surg Med. 2010;42(2):113-122.

15. Cavalie M, Sillard L, Montaudie H, Bahadoran P, Lacour JP, Passeron T. Treatments of keloids with laser-assisted topical steroid delivery: a retrospective study of 23 cases. Dermatol Ther. 2015;28(2):74-78.

16. Ma G, Wu P, Lin X, et al. Fractional carbon dioxide laser-assisted drug delivery of topical timolol solution for the treatment of deep infantile hemangioma: a pilot study. Pediatr Dermatol. 2014;31(3):286-291.

17. Waibel J, Wulkan A. Split face comparison of the effects of vitamin CE ferulic formula serum to decrease post-operative recovery and increase neocollagenesis in fractional ablative laser resurfacing for photodamage. Laser Surg Med. 2013;45(25):1-93.

18. Lee WR, Shen SC, Al-Suwayeh SA, Yang HH, Yuan CY, Fang JY. Laserassisted topical drug delivery by using a low-fluence fractional laser: Imiquimod and macromolecules. J Control Release. 2011;153(3):240-248.

19. Hsiao CY, Huang $\mathrm{CH}, \mathrm{Hu} \mathrm{S}$, et al. Fractional carbon dioxide laser treatment to enhance skin permeation of ascorbic acid 2-glucoside with minimal skin disruption. Dermatol Surg. 2012;38(8):1284-1293.

20. Budamakuntla L, Loganathan E, Suresh DH, et al. A randomised, open-label, comparative study of tranexamic acid microinjections and tranexamic acid with microneedling in patients with melasma. J Cutan Aesthet Surg. 2013;6(3):139-143.

21. Baron ED, Harris L, Redpath WS, et al. Laser-assisted penetration of topical anesthetic in adults. Arch Dermatol. 2003;139(10):1288-1290.

22. Attwa E, Khater M, Assaf M, Abdel Haleem M. Melasma treatment using an erbium:YAG laser: a clinical, immunohistochemical, and ultrastructural study. Int J Dermatol. 2015;54(2):235-244.

23. Lee WR, Shen SC, Kuo-Hsien W, Hu CH, Fang JY. Lasers and microdermabrasion enhance and control topical delivery of vitamin C. J Invest Dermatol. 2003;121(5):1118-1125.

24. Lukac M, Vizintin Z, Kazic M, Sult T. Novel fractional treatments with VSP erbium YAG aesthetic lasers. J Laser Health Acad. 2008;6(1):1-12.
Clinical, Cosmetic and Investigational Dermatology

\section{Publish your work in this journal}

Clinical, Cosmetic and Investigational Dermatology is an international, peer-reviewed, open access, online journal that focuses on the latest clinical and experimental research in all aspects of skin disease and cosmetic interventions. This journal is included on PubMed. The manuscript management system is completely online

\section{Dovepress}

and includes a very quick and fair peer-review system, which is all easy to use. Visit http://www.dovepress.com/testimonials.php to read real quotes from published authors 